\title{
Semaphorin 3E/PlexinD1 signaling is required for cardiac ventricular compaction
}

\author{
Reddemma Sandireddy, ${ }^{1}$ Dasan Mary Cibi, ${ }^{1}$ Priyanka Gupta,, ${ }^{1}$ Anamika Singh, ${ }^{1}$ Nicole Tee, ${ }^{2}$ \\ Akiyoshi Uemura, ${ }^{3}$ Jonathan A. Epstein, ${ }^{4}$ and Manvendra K. Singh ${ }^{1,2}$ \\ 1Program in Cardiovascular and Metabolic Disorders, Duke-NUS Medical School Singapore, Singapore. ${ }^{2}$ National Heart \\ Research Institute Singapore, National Heart Center Singapore, Singapore. ${ }^{3}$ Department of Retinal Vascular Biology, \\ Nagoya City University Graduate School of Medical Sciences, Mizuho-ku, Nagoya, Japan. ${ }^{4}$ Penn Cardiovascular Institute, \\ Department of Medicine, Department of Cell and Developmental Biology, and Institute for Regenerative Medicine, \\ Perelman School of Medicine at the University of Pennsylvania, Philadelphia, Pennsylvania, USA.
}

\begin{abstract}
Left ventricular noncompaction (LVNC) is one of the most common forms of genetic cardiomyopathy characterized by excessive trabeculation and impaired myocardial compaction during fetal development. Patients with LVNC are at higher risk of developing left/right ventricular failure or both. Although the key regulators for cardiac chamber development are well studied, the role of semaphorin (Sema)/plexin signaling in this process remains poorly understood. In this article, we demonstrate that genetic deletion of PIxnd1, a class-3 Sema receptor in endothelial cells, leads to severe cardiac chamber defects. They were characterized by excessive trabeculation and noncompaction similar to patients with LVNC. Loss of PIxnd1 results in decreased expression of extracellular matrix proteolytic genes, leading to excessive deposition of cardiac jelly. We demonstrate that PIxnd1 deficiency is associated with an increase in Notch1 expression and its downstream target genes. In addition, inhibition of the Notch signaling pathway partially rescues the excessive trabeculation and noncompaction phenotype present in PIxnd1 mutants. Furthermore, we demonstrate that Semaphorin 3E (Sema3E), one of PlexinD1's known ligands, is expressed in the developing heart and is required for myocardial compaction. Collectively, our study uncovers what we believe to be a previously undescribed role of the Sema3E/PlexinD1 signaling pathway in myocardial trabeculation and the compaction process.
\end{abstract}

Conflict of interest: The authors have declared that no conflict of interest exists.

Copyright: @ 2019 American Society for Clinical Investigation

Submitted: October 30, 2018

Accepted: May 1, 2019

Published: June 20, 2019.

Reference information: /Cl Insight. 2019;4(12):e125908. https://doi. org/10.1172/jici.insight.125908.

\section{Introduction}

Myocardial trabeculation and compaction are the most important and complex processes involved in ventricular chamber maturation. Both processes require delicate spatial and biochemical interactions among various cell types. In mice, myocardial trabeculation was proposed to start after cardiac looping at E9.0 (1, 2). However, a recent study suggests that it begins earlier during the linear heart tube formation stage (E8.0) $(3,4)$. During trabeculation, myocardial cells protrude into the extracellular cell matrix (ECM), also referred to as cardiac jelly, and form a network of luminal projections called trabeculae (1, 2). Trabeculae consist of myocardial cells covered by a layer of endocardial cells (5). The endocardial cells send signals to the underlying cardiomyocytes to migrate, proliferate, and form the trabeculae in the developing heart. This process increases the surface area and improves nutrition and oxygen uptake in the myocardium prior to coronary vascularization $(1,5)$. As the cardiac development progresses, trabeculae undergo significant compaction that transforms them from spongy to compact myocardium (1, 6). The compact layer then grows and subsequently provides a major contractile force required to drive circulation (7-9). Defects in the chamber maturation process may lead to various cardiac disorders. One of the most characterized disorders of the cardiac chamber maturation is left ventricular noncompaction (LVNC). This condition is characterized by prominent ventricular trabeculae, a thin myocardium, and deep intertrabecular recesses among the trabeculae (10-13). Although LVNC mainly affects the left ventricle, isolated right ventricular or biventricular noncompaction has also been reported (14-16). Clinically, LVNC is associated with left ventricular dilation, hypertrophy, or other forms of congenital cardiac 
diseases. Patients with LVNC are at a higher risk for developing systolic and diastolic dysfunction leading to heart failure, arrhythmias, and sudden cardiac death (17-19).

The underlying molecular and cellular mechanisms that orchestrate ventricular trabeculation and compaction are governed by various transcription factors (e.g., Nkx2.5, T-box transcription factors, Gata4 and 6, Irx3 and 5, etc.) and signaling pathways (e.g., Notch, Neuregulin [Nrg1], Ephrin, ErbB, Bmp, etc.) (20). Genetic mutations in these genes lead to defects in cardiac chamber development and maturation including LVNC (21). Notch signaling is one of the most studied pathways in this process (22). Notch1 is expressed in the endocardial cells near the proximal end of the trabecular myocardium, where it controls the ventricular trabeculation and compaction by regulating Bmp10 signaling and $\operatorname{Nrg} 1$ expression $(3,23)$. Bmp10 is a cytokine and member of TGF- $\beta$ superfamily. It is expressed as early as E9.5 in the trabecular myocardium and promotes cardiomyocyte proliferation (24). Although Bmp10 deletion leads to defective cardiomyocyte proliferation resulting in impaired ventricular trabeculation, its overexpression results in cardiac hypertrabeculation and noncompaction $(24,25)$. However, the exact molecular link between Notch signaling pathways and Bmp10 is yet to be elucidated. In contrast, the signaling pathway between Notch1 and Nrg1 is well established. Notch1 inhibits the transcriptional activation of $\mathrm{Nrg} 1$ by binding to its promoter region. This process affects the $\mathrm{Nrg} 1 / \mathrm{ErbB}$ signaling pathway required for proliferation and differentiation of ventricular myocardial cells. Genetic deletion of Notch1 or its transcriptional cofactor Rbpjk within the endothelial cells results in hypotrabeculation (3, 23). In contrast, upregulation of Notch1 in endocardial cells leads to ventricular hypertrabeculation and noncompaction (26-29).

The cardiac jelly located between the endocardium and myocardium facilitates this orchestration of molecules among the cardiac layers. It serves as a substrate for cell migration and diffusion of signaling molecules expressed in the 2 cardiac layers $(4,27,30,31)$. Synthesis and degradation of this ECM is a notable feature of cardiac remodeling. Although its synthesis is crucial for the above-mentioned molecular communication between the 2 cardiac layers, its degradation is essential for myocardial compaction (31). Thus, a fine balance between the synthesis and degradation of ECM components is required for proper regulation of trabeculation and the myocardial compaction process. Many studies have demonstrated that impaired ECM synthesis or degradation can cause trabeculation and compaction defects (4, 27, 32-36). Genetic deletions of genes required for cardiac jelly formation and degradation lead to hypotrabeculation and hypertrabeculation/noncompaction, respectively. Despite the essential role of ECM in trabeculation and compaction, pathways that control ECM dynamics remain poorly understood.

Semaphorins (Semas) are a large family of secreted or membrane-associated glycoproteins (37). Semas bind to either plexin receptors or the plexin-neuropilins (NRP1/2) receptor complex to transduce their signal. While the majority of the membrane-associated Semas bind to the plexin receptors directly, most of the secreted Semas (except Sema3E) require NRP1 or NRP2 to transmit their signal to plexin receptors $(37,38)$. Impaired Sema/plexin signaling is associated with many cardiovascular defects, such as persistent truncus arteriosus, sinus bradycardia, ventricular septal defects, anomalous pulmonary venous return, and coronary and aortic arch defects $(37,39-43)$. However, the role of the Sema/plexin pathway in ventricular trabeculation and compaction has not been well established. The only Sema implicated in this process is Sema6D. Sema6d is expressed in both trabecular and compact myocardial cells, but its receptor Plxnal expression is restricted to the trabecular myocardial and endocardial cells. A study by Toyofuku et al. demonstrated that knockdown of either Sema6d or Plxnal or both in chick embryos results in a noncompaction phenotype. This phenotype is characterized by a thin compact and poorly trabeculated myocardium, suggesting that Sema6D/plexinA1 interaction is significant in cardiac chamber development (44). However, both Sema6dand Plxna1-knockout mice are viable and show no obvious cardiac defects (45-47). Hence, in-depth investigations are necessary to elucidate the role of Sema/plexin signaling in cardiac chamber development.

In the current study, we focus on PlexinD1, another Sema receptor. Plxnd1 is expressed in the embryonic endothelial cells including the endocardium and is maintained throughout development (48-50). Unlike other Semas, Sema3E, a known ligand of PlexinD1, binds directly to PlexinD1 to transmit signals to the downstream effectors (38). Previously, Sema3E/PlexinD1 signaling has been elegantly demonstrated to regulate axonal growth and vascular formation (51). Although the expression and function of Sema3E in cardiac development has not been reported, a role for PlexinD1 in cardiovascular development was revealed with the identification of cardiac outflow tract (truncus arteriosus) and other vascular defects in Plxnd1-knockout embryos $(49,50)$. Truncus arteriosus has been associated with LVNC in various murine models, suggesting a possible role of PlexinD1 in the LVNC phenotype $(29,52,53)$. However, the molecular mechanism of PlexinD1 signaling regulating myocardial trabeculation and compaction is unclear. 
In this article, we demonstrate that PlexinD1 signaling is essential for ventricular trabeculation and compaction. Global or endothelial-specific deletion of Plxnd1 leads to ventricular hypertrabeculation and noncompaction. Loss of Plxnd1 results in decreased expression of gene-encoding proteases that degrade ECM, leading to excessive deposition of cardiac jelly. Molecular analyses demonstrate that Notch pathway genes are upregulated in Plxnd1-knockout hearts. Inhibition of Notch signaling partially rescues the hypertrabeculation and noncompaction phenotypes observed in $P l x n d 1^{-1-}$ hearts. Sema3E is also expressed in the developing heart and required for proper ventricular compaction. These results indicate that the Sema3E/ PlexinD1 signaling pathway is required for inhibiting the Notch signaling pathway during trabeculation and compaction. Together, they suggest a novel role of the Sema3E/PlexinD1 signaling pathway in ventricular chamber formation and maturation.

\section{Results}

Plxnd1 deletion results in ventricular hypertrabeculation and noncompaction. Plxnd1-knockout mice die after birth and display cardiovascular abnormalities, including aortic arch anomalies, persistent truncus arteriosus, ventricular septal defects, and a decrease in ventricular wall thickness (49). Because ventricular trabeculation and myocardial compaction are essential for a fully functional and competent ventricular wall, we investigated whether thinning of the ventricular wall in $P l x n d 1^{-/-}$hearts was due to impaired trabeculation and compaction. We measured both the trabecular and compact layer thickness from E10.5 to E15.5 embryos. These embryonic stages were chosen because at E10.5, cardiomyocytes along the inner wall protrude into the ventricular lumen to form trabecular myocardium, while the outer myocardial cells become organized and form the compact myocardium. By E15.5, this process is completed and the majority of the trabecular myocardium becomes compact. No significant difference in the thickness of the trabecular or compact layer was observed between E10.5 control and Plxnd1 ${ }^{-/-}$embryos (Figure 1, A-C). However, histological analysis of E12.5, E14.5, and E15.5 embryos revealed myocardial trabeculation and compaction

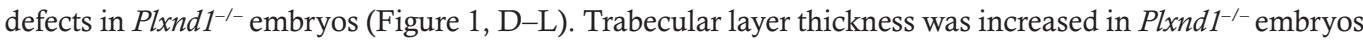
in comparison to their control littermates, indicating a hypertrabeculation phenotype (Figure 1, D-L). The

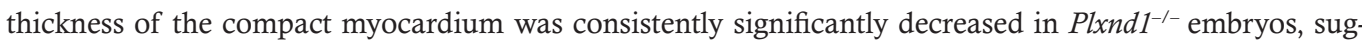
gesting a noncompaction phenotype (Figure 1, D-L).

Endothelium-specific deletion of Plxnd1 (Plxnd $1^{e K O}$ ) recapitulates hypertrabeculation and noncompaction defects observed in Plxnd1 $1^{-1}$ mice. PlexinD1 is expressed in the embryonic endothelial cells including the endocardium and is maintained throughout development (Supplemental Figure 1; supplemental material available online with this article; https://doi.org/10.1172/jci.insight.125908DS1). In order to determine the tissue-specific functions of PlexinD1 accountable for the hypertrabeculation and noncompaction defects observed in Plxnd1 $1^{-1-}$ mice, we generated endothelium-specific Plxnd1-knockout $\left(P l x n d 1^{e K O}\right)$ mice using the Tie2-Cre line. Tie2 is expressed as the first endothelial cells arise, and its expression is maintained in all endothelial cells throughout embryonic and adult life. We performed histological and quantitative analyses of Plxnd1 $1^{\text {KOO }}$ hearts from E10.5, E12.5, and E15.5 embryos (Figure 2). At E10.5, no obvious difference in the thickness of the trabecular or the compact layer was observed between control and Plxnd1 ${ }^{\text {eKO }}$ embryos (Figure 2, A-C). On the contrary, at later embryonic stages (E12.5 and E15.5), Plxnd1 ${ }^{\text {eKO }}$ embryos exhibited hypertrabeculation and noncompaction defects, similar to those in Plxnd1 $1^{-1}$ embryos (Figure 2, D-I). Compared with control littermates, the compact myocardium thickness was significantly reduced, while the thickness of the trabecular layer was significantly increased in Plxnd1 ${ }^{\text {eKO }}$ embryos (Figure 2, D-I).

Increased Bmp10 signaling and cardiomyocyte proliferation in Plxnd1 $1^{-1-}$ mice. We analyzed Bmp10 expression in both Plxnd1 $1^{--}$and control hearts. Next, we performed quantitative PCR (qPCR), immunohistochemistry, and Western blot experiments using hearts isolated from control and Plxnd1 ${ }^{-1-}$ embryos. In comparison to

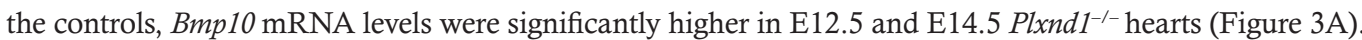
Both Western blot and immunohistochemistry analyses show that an increase in Bmp10 transcript correlates with its protein level in Plxnd1 ${ }^{-/-}$hearts (Figure 3, B and C, and Supplemental Figure 4A). To assess whether Bmp10 upregulation affected myocardial cell proliferation in Plxnd1 ${ }^{-/-}$embryos, we also performed Ki-67 and MF20 double immunostaining on cardiac sections from E14.5 embryos. A significant increase of Ki-67

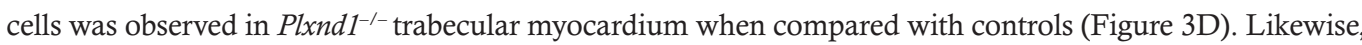
expression of other trabecular myocardial and endocardial markers such as Irx3, Irx5, and PEG1 was also increased in $P l x n d 1^{-1-}$ hearts (Supplemental Figure 2A). However, expression of cardiac chamber regulators such as Hopx, Cx40,Cx43, Tbx5, and Nkx2.5 was not affected by Plxnd1 deletion (Supplemental Figure 2B). 

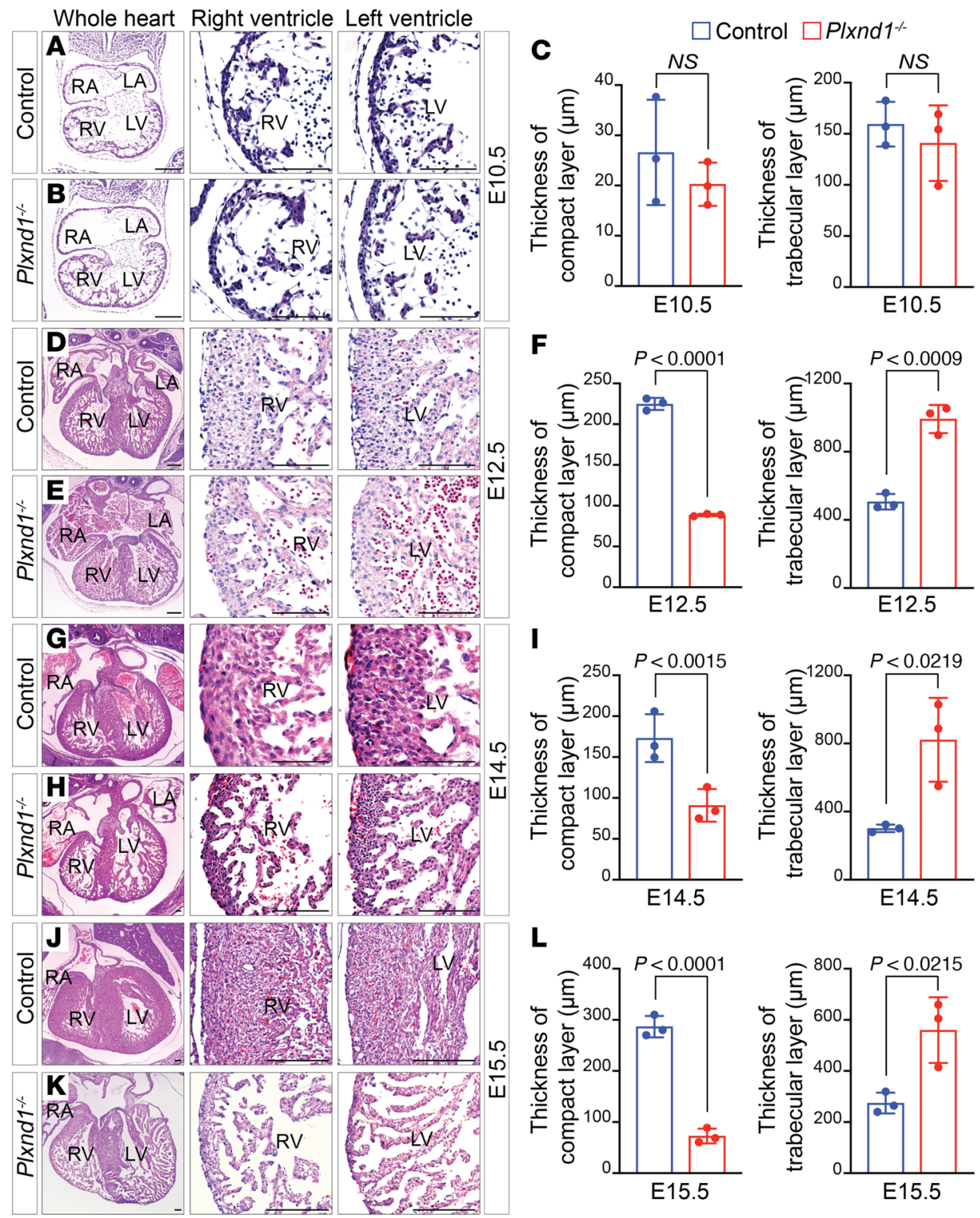

$\mathbf{L}$
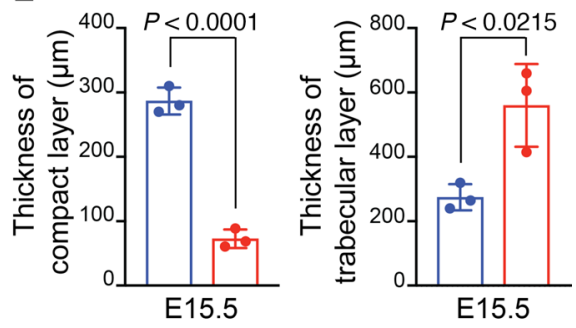

Figure 1. PIxnd1-knockout mice develop LVNC. H\&E staining of paraffin sections of E10.5 (A and B), E12.5 (D and E),

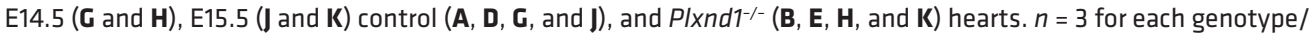
time point. Quantification of the thickness of the compact and trabecular layer in control $(n=3)$ and Plxnd1 $1^{-/-}$hearts $(n$ $=3$ ) at different time points are indicated as $\mathbf{C}, \mathbf{F}, \mathbf{I}$, and $\mathbf{L}$. LA, left atrium; LV, left ventricle; RA, right atrium; RV, right ventricle. Scale bars: $100 \mu \mathrm{m}$ (A, B, D, E, G, and H); $200 \mu \mathrm{m}$ (J and K).

Plxnd1 deletion impairs ECM dynamics in the developing heart. To determine the dynamics of ECM synthesis and degradation and its potential role in ventricular trabeculation and the compaction process, we harvested E11.5, E12.5, and E14.5 hearts from the WT embryos and performed gene expression analysis (Supplemental Figure 3). These embryonic time points were selected because Plxnd1 mutants develop severe hypertrabeculation and noncompaction defects during these stages. We observed that ECM proteolytic genes such as Adamts-1, -5, -7, -15, and -19 were initially expressed at lower levels in E11.5 hearts of WT embryos, but their expression increased as the embryos developed (Supplemental Figure 3A). In contrast, ECM synthesis genes such as hyaluronan synthase-2 (Has2) and versican (Vcan) were initially expressed at high levels at E11.5 but decreased as they developed (Supplemental Figure 3B).

We next checked the effect of Plxnd1 deletion on ECM synthesis and degradation. Expression analysis on ECM proteolytic genes, such as Adamts-1, -5, -7, -15, Hyal2, and Mmp2 show significant downregulation of these genes in Plxnd1 $1^{-/}$hearts (Figure 4A). No significant change in Adamts19 expression was observed. 

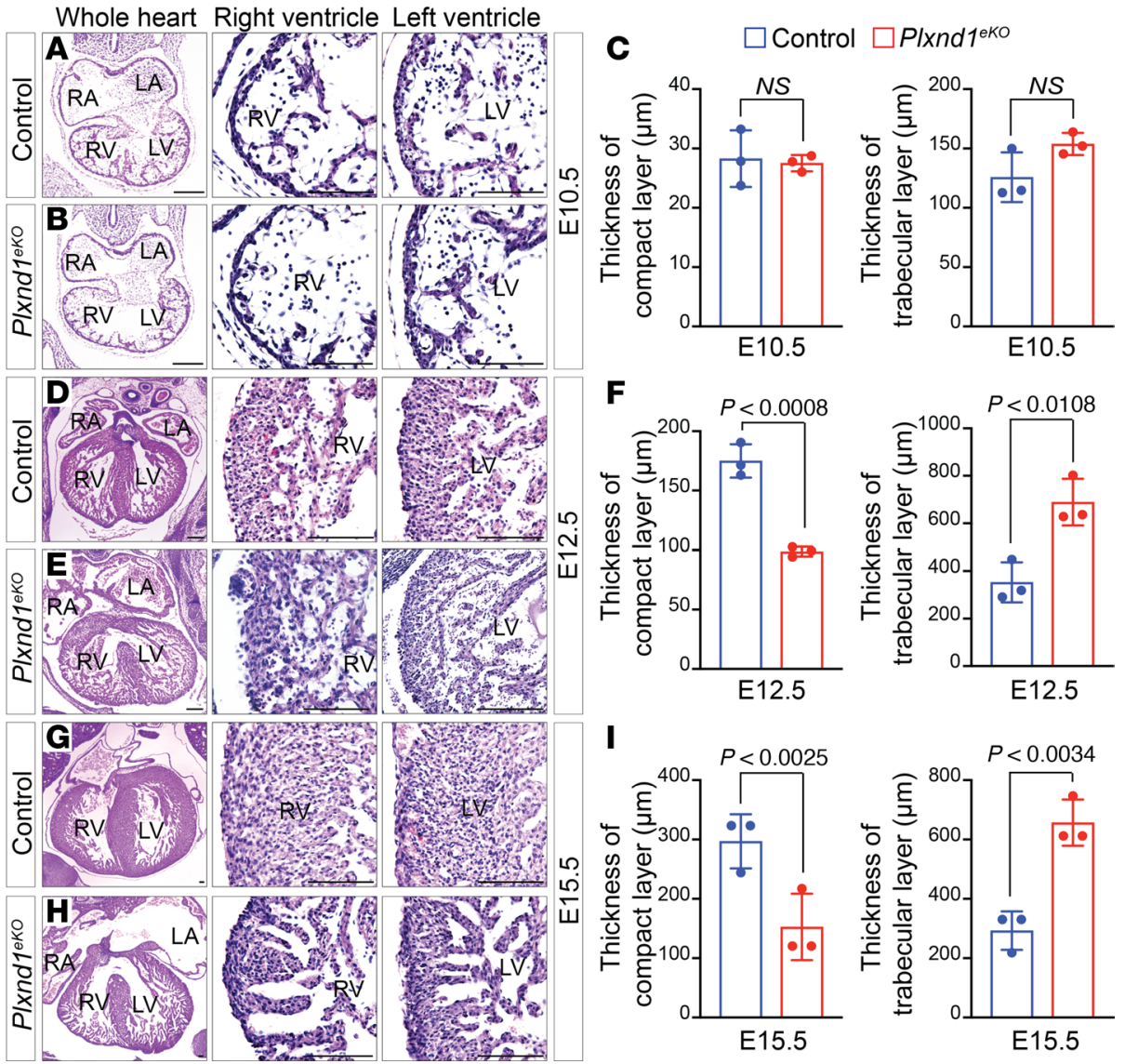

Figure 2. Endothelial-specific deletion of PIxnd1 (PIxnd ? $^{\text {kO }}$ ) results in LVNC. H\&E staining of paraffin sections of E10.5 (A and B), E12.5 (D and E), and E15.5 ( $\mathbf{G}$ and $\mathbf{H})$ control (A, D, and $\mathbf{G})$ and PIxnd ${ }^{\mathrm{kO} O}$ (B, E, and $\mathbf{H}$ ) hearts. $n=3$ for each genotype/time point. Quantification of the thickness of the compact and trabecular layer in control $(n=3)$ and PIxnd ${ }^{\text {кKo }}$ hearts $(n=3)$ at different time points are indicated as C, F, and I. LA, left atrium; LV, left ventricle; RA, right atrium; RV, right ventricle. Scale bars: $100 \mu \mathrm{m}$ (A, B, D, E, G, and H).

In comparison to proteolytic genes, expressions of ECM synthesis genes such as Has2, CD44 (a hyaluronan receptor), and Vcan were not significantly different between control and $P l x n d 1^{-1-}$ hearts (Figure $\left.4 \mathrm{~B}\right)$. Consistent with the dynamic expression of ECM proteolytic genes, intensity of Alcian blue staining in E11.5

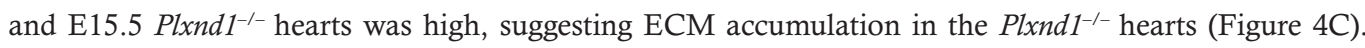
Immunostaining for versican also recapitulated the Alcian blue staining in Plxnd1 $1^{-/-}$hearts (Figure 4D). We also observed increased protein expression of versican and fibronectin and decreased expression of Adamts1, suggesting ECM accumulation in the Plxnd1 $1^{-{ }^{-}}$hearts (Figure 4E and Supplemental Figure 4D).

Upregulation of Notch and Nrg1 signaling pathways in Plxnd1 $1^{-/-}$hearts. To investigate the molecular communication between the endocardium and myocardium, key genes of the Notch and the Nrg1 pathway involved in chamber development were examined. Notch1, Hrt1, Hrt2, Hrt3, EphrinB2, EphrinB4, Nrg1, ErbB2, and ErbB4 in both control and Plxnd1 ${ }^{-/}$hearts at E10.5, E12.5, and E14.5 embryonic stages were analyzed by qPCR. At E10.5, we observed increased expression of only Notch1, Hrt2, EphrinB4, and Nrg1 in Plxnd1 $1^{-1-}$ hearts (Figure 5, A and B). However, at later time points, most of the Notch pathway genes were significantly upregulated (Figure 5, A and B). We performed Western blot analysis in E12.5 and E14.5 heart extracts from control and mutant embryos to determine whether changes in RNA transcripts were correlated to their protein levels. A significant increase in Notch1 expression was observed in Plxnd1 ${ }^{-1-}$ hearts (Figure 5C and Supplemental Figure 4B). Consistent with increased expression of Notch1 genes,

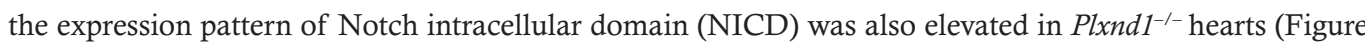
5C). Immunostaining for NICD showed enhanced Notch1 activity in Plxnd1 mutant hearts. (Figure 5D). Notch1 signaling is required for the expression of the ephrins (Eph-B2/B4), which in turn are necessary for Nrg1/ErbB2 signaling. We next determined the effect of the upregulated Notch pathway on Nrg1/ErbB2 
A

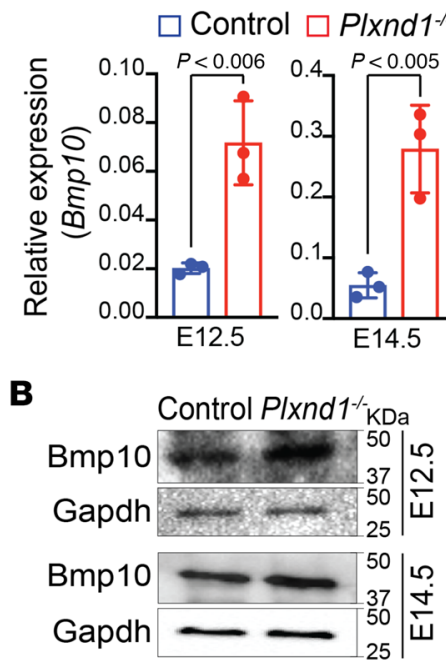

C
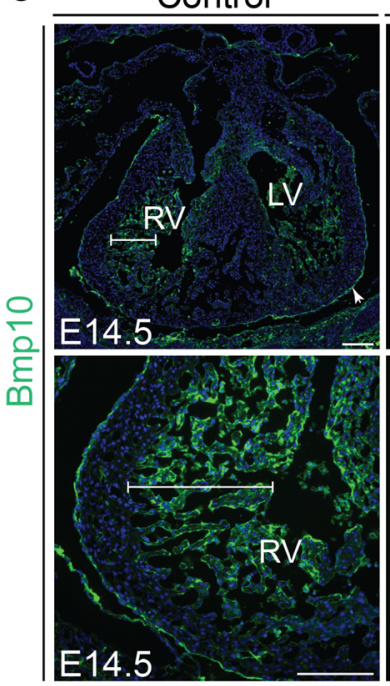

Plxnd1 $1-$

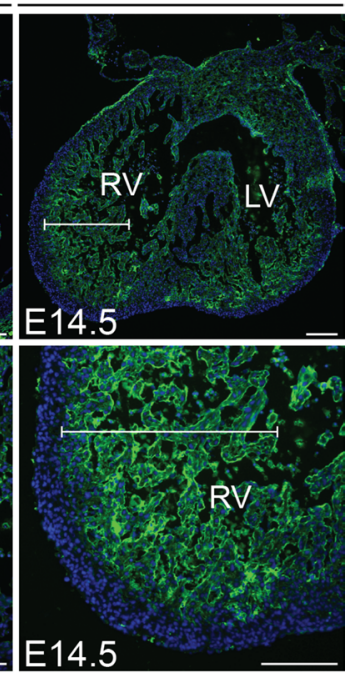

$\square$ Control $\square$ PIxnd1\%

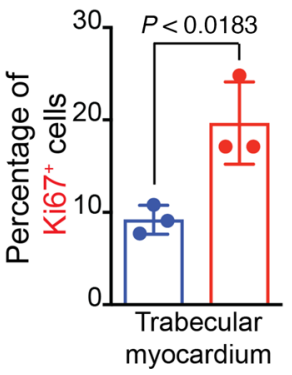

myocardium

D

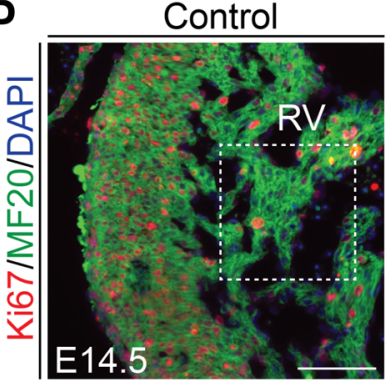

Plxnd1\%

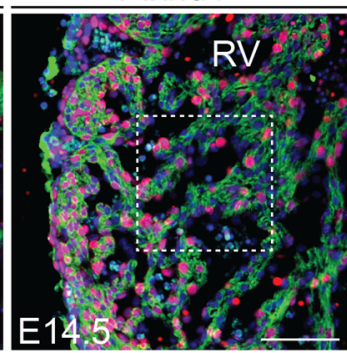

Figure 3. Upregulation of Bmp10 in PIxnd1 1/- hearts. Real-time qPCR analysis of Bmp10 using RNA isolated from control and Plxnd1 $1^{-/}$hearts. $n=3$ for each time point. (A). Representative Western blot demonstrating induction of Bmp10 expression in E12.5 and E14.5 Plxnd1 1/- hearts (B). Gapdh was used as a loading control. Immunostaining for Bmp10 was performed on E14.5 control and PIxnd1 1- heart sections (C). White lines represent Bmp10-positive trabecular myocardium (C). Double immunostaining for Ki-67 and MF20 on heart transverse sections obtained from E14.5 control and Plxnd1 ${ }^{-1-}$ embryos. Quantification of cell proliferation was calculated as the ratio of Ki-67-positive cells to the total number of cells as determined by DAPI counterstain in the defined area of trabecular myocardium in control and PIXnd1 1/- hearts (D). $n=3$ for each genotype. LV, left ventricle; RV, right ventricle. Scale bars: $100 \mu \mathrm{m}$.

signaling. Western blot analysis showed that both ErbB2 and pErbB2 levels were upregulated in the Plxnd1 mutant hearts at E12.5 and E14.5 (Figure 5E and Supplemental Figure 4C).

We hypothesize that the Notch pathway was activated in the observed hypertrabeculated and noncompacted ventricles. Thus, reducing Notch1 activation in vivo may rescue these defects in Plxnd1 $1^{-1-}$ hearts. We treated pregnant mice at E11.5 with $N$-[(1S)-2-[[(7S)-6,7-Dihydro-5-methyl-6-oxo-5H-dibenz[b,d] azepin-7-yl] amino]-1-methyl-2-oxoethyl]-3,5-difluorobenzeneacetamide (DBZ), a $\gamma$-secretase inhibitor, to inhibit Notch signaling in the embryos. The embryos were harvested at E13.5 for histological analysis (Figure 5F). The compact myocardium thickness in DBZ-treated $P l x n d 1^{-/-}$embryos was significantly increased in vehicle-treated $P l x-$ $n d 1^{-1-}$ embryos (Figure 5G). Conversely, the trabecular myocardium thickness was significantly reduced in the DBZ-treated Plxnd1 $1^{-1-}$ embryos (Figure 5G). Furthermore, gene expression analysis demonstrated that partial rescue of hypertrabeculation and noncompaction phenotypes in Plxnd1 $1^{-/-}$embryos after DBZ treatment was associated with reduced expression of Notch1 and Bmp10 and increased expression of Adamts15 (Figure 5H).

Plxnd1 overexpression does not affect cardiac chamber development but can rescue the hypertrabeculation and noncompaction phenotype when crossed to the Plxnd1-null background. To specifically explore the role of Plxnd1 in the endothelium during myocardial trabeculation and compaction, we generated endothelial-specific Plxnd1-transgenic mice using a murine tyrosine kinase receptor Tie2 promoter (Tie2-Plxnd1 Tg; Figure 6A). Founders were genotyped by transgene-specific PCR (Figure 6B). Overexpression of PlexinD1 in transgenic embryos was validated in Western blot analysis of protein extracts isolated from E10.5 embryos. We also isolated endothelial cells from control and transgenic embryos and performed qPCR analysis for Plxnd1. As 
A
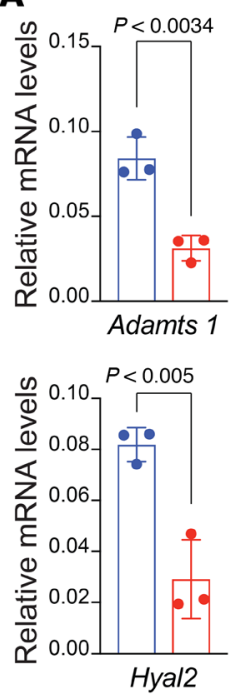

C

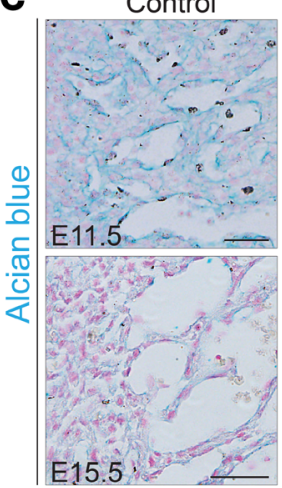

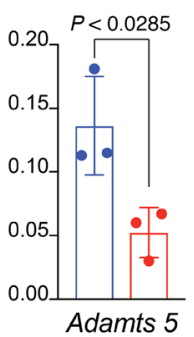

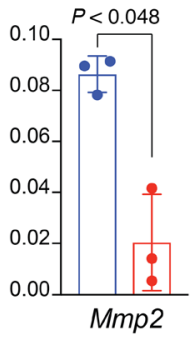

Plxnd1

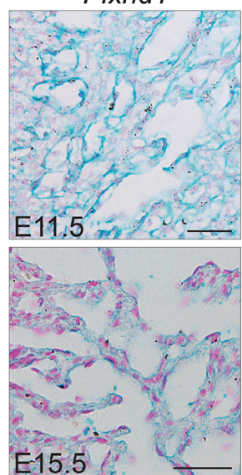

$\square$ Control $\square$ Plxnd1
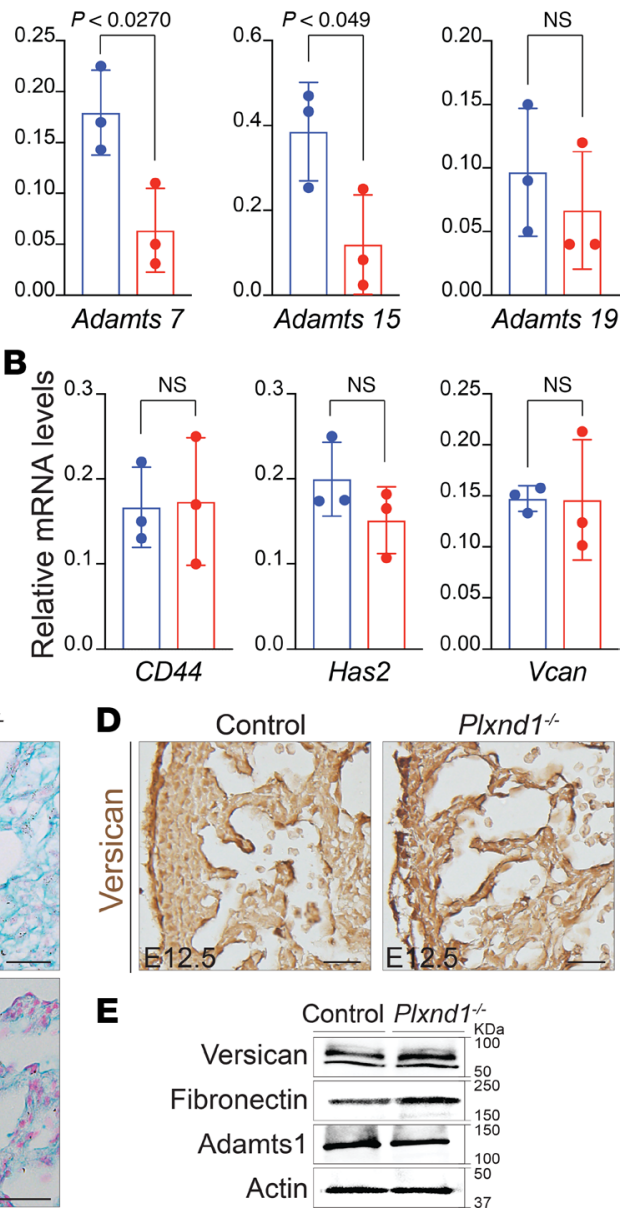

Figure 4. PIxnd1 deletion leads to impaired ECM dynamics in the developing heart. Real-time qPCR analysis of ECM proteolytic and synthesis genes using RNA isolated from E11.5 control and Plxnd1 $1^{-/-}$hearts. $n=3$ for each genotype (A and B). Alcian blue staining for extracellular matrix and counterstain with Nuclear Fast Red solution was performed on E11.5 and E15.5 cardiac sections (C). Immunohistochemistry for versican on E12.5 control and P/xnd1 ${ }^{-/-}$heart sections (D). Representative Western blot analysis of versican, fibronectin, and Adamts1 using E14.5 control and Plxnd1 ${ }^{-/-}$heart lysates. Actin was used as loading control (E). Scale bars: $100 \mu \mathrm{m}$.

expected, both PlexinD1 mRNA and protein levels were elevated in the transgenic embryos (Figure 6, C and D). To evaluate whether overexpression of endothelial-specific Plxnd1 influenced myocardial trabeculation and the compaction process, we analyzed the control and Tie2-Plxnd1 Tg hearts isolated from E12.5 and E14.5 embryos. Compared with control littermates, no obvious difference in either trabecular or compact ventricular myocardial layer thickness was observed in Tie2-Plxnd1 Tg embryos at various time points (Figure 6E). We further investigated the effect of endothelial-specific restoration of Plxnd1 on hypertrabeculation and noncompaction defects observed in $P l x n d 1^{-1-}$ mice. We crossed transgenic mice overexpressing Plxnd1 in the endothelial cells (Tie2-Plxnd1 Tg) with the Plxnd1 ${ }^{+/}$line to generate Tie2-Plxnd1 Tg Plxnd1 $1^{+-}$ mice. These mice were then crossed with Plxnd1 $1^{+/}$to generate Tie2-Plxnd1 Tg Plxnd1 $1^{-/-}$embryos. When Tie2-Plxnd1 Tg mice were crossed to a Plxnd1-null background, the trabecular layer thickness decreased while the compact myocardial layer increased (Figure 6F).

Sema3E is expressed in the heart and required for myocardial trabeculation and compaction. We established Sema3E expression to further discern its role in the developing heart. Cardiac sections from E12.5 and E14.5 embryos were immunostained with Sema3E antibody. In contrast to endothelium-specific PlexinD1 expression, Sema3E expression was more broadly expressed in the developing heart. Many different cardiac cell types including endocardial, cardiac valve, cardiomyocytes, and epicardium expressed Sema3E (Figure 7, A and B). Sema3E-alkaline phosphatase (AP) binding experiments performed on E15.5 embryo cryosections revealed strong Sema3E-AP binding in the neural tube, lungs, cartilage, and heart (Figure 7C). 
A
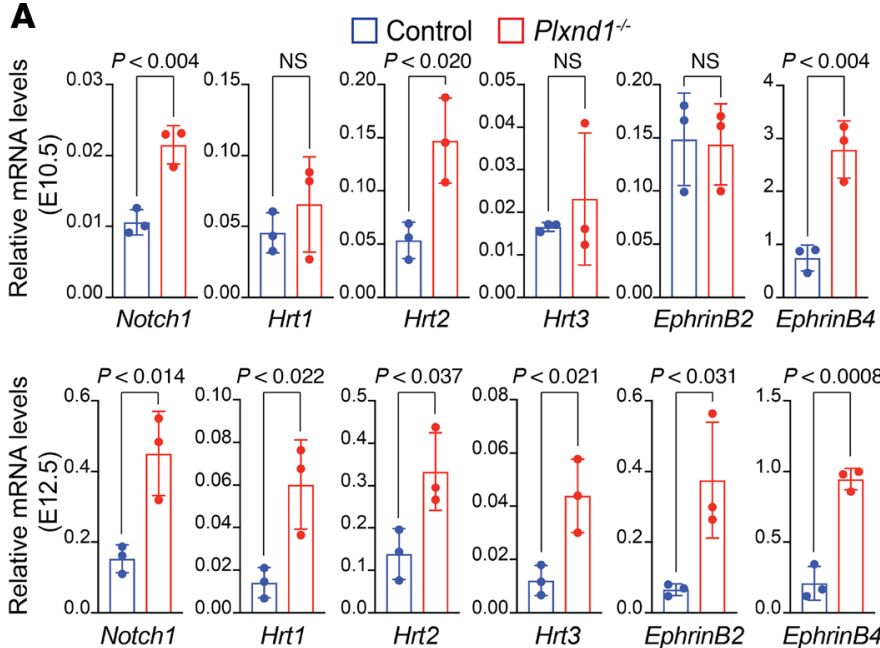

$P<0.031 \quad P<0.0008$
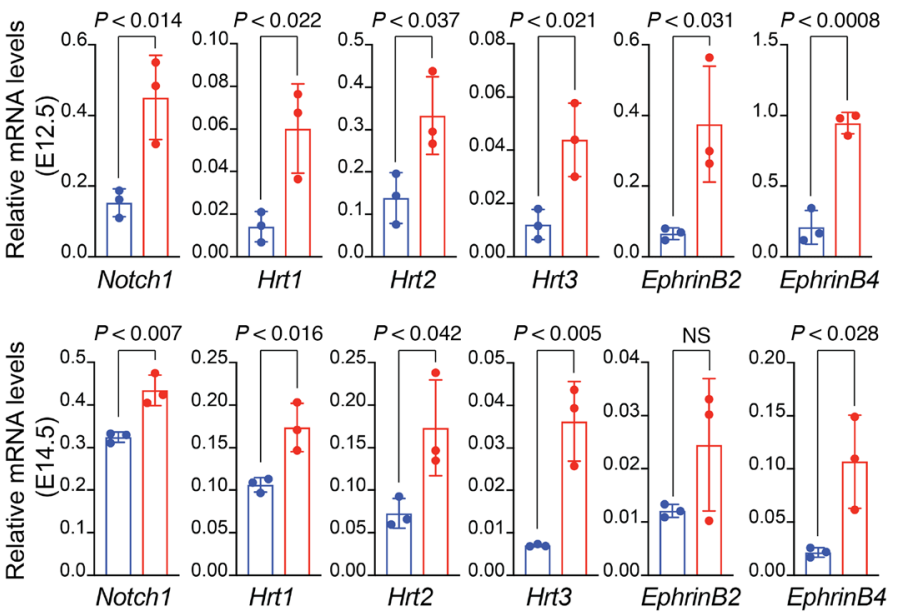

C

E12.5

E14.5

Control Plxnd1 1 Control Plxnd1
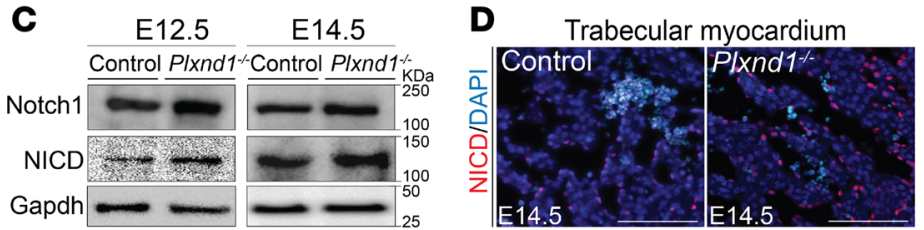

B
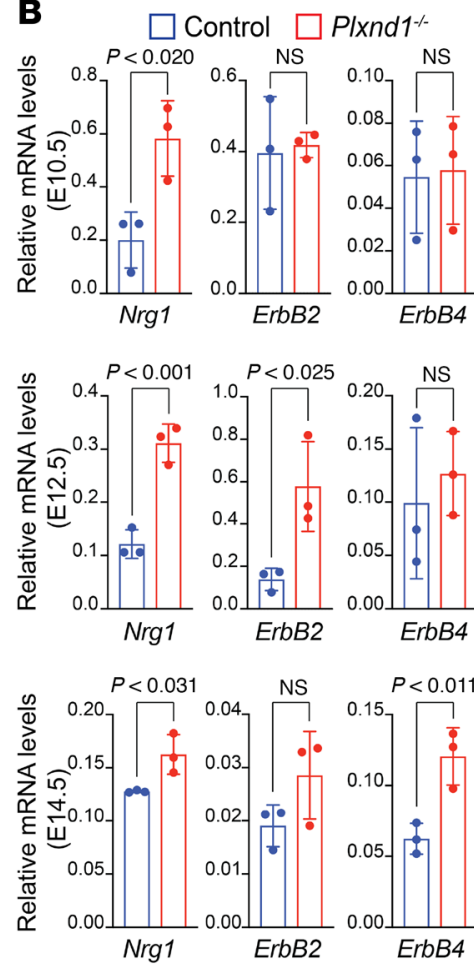

E

E12.5 E14.5 Control Plxnd1 ${ }^{-}$Control Plxnd1 ${ }^{-}$KDa

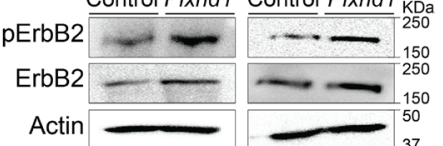

$\mathbf{F}$

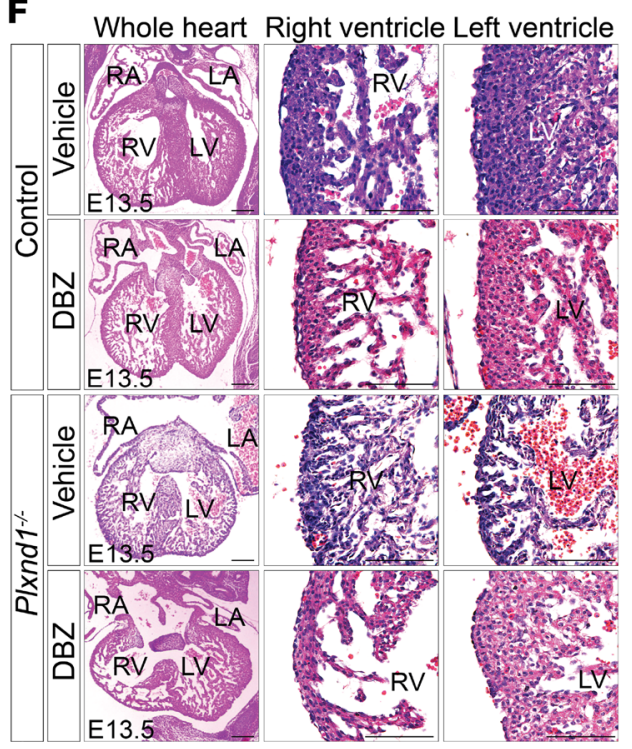

G

\begin{abstract}
Control + Vehicle Plxnd1 1 + Vehicle
Control + DBZ Plxnd1/ + DBZ
\end{abstract}
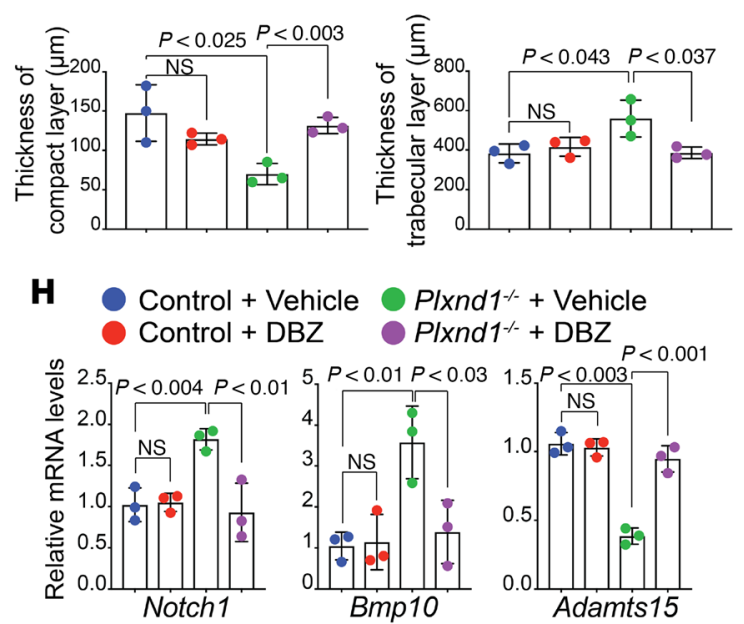

$1^{-}+$Vehicle

$d 1^{-\tau}+\mathrm{DBZ}$
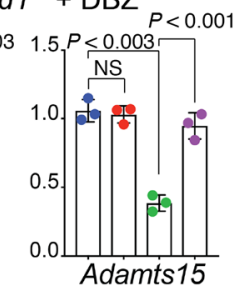

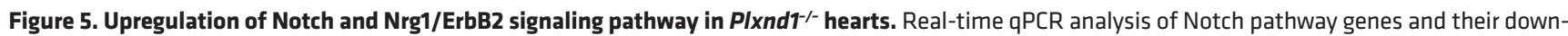
stream targets using RNA isolated from E10.5, E12.5, and E14.5 control and Plxnd1 ${ }^{-1-}$ hearts (A). Expression levels of Nrg1, ErbB2, and ErbB4, as measured by qPCR using RNA isolated from E10.5, E12.5, and E14.5 control and Plxnd1 1/- hearts. $n=3$ for each genotype/time point (B). Representative Western blot of Notch1 and NICD on E12.5 and E14.5 control and PIxnd1 1/- heart lysates. Gapdh was used as loading control (C). Immunostaining for NICD on E14.5 control

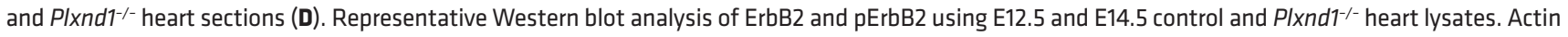
was used as loading control (E). Partial rescue of hypertrabeculation and noncompaction defects displayed by Plxnd1 $7^{-/-}$embryos after treatment with the $\gamma$-secretase inhibitor DBZ. H\&E staining of E13.5 control and PIXnd1 $1^{-/-}$hearts isolated after vehicle or DBZ treatment (F). Quantification of the thickness of the compact and trabecular layer (C). $n=3$ for each genotype. Real-time qPCR analysis of Notch1, Bmp10, and Adamts15 using RNA isolated from E13.5 control and PIxnd1 ${ }^{-1-}$ hearts isolated after vehicle or DBZ treatment (H). LA, left atrium; LV, left ventricle; RA, right atrium; RV, right ventricle. Scale bars: $100 \mu \mathrm{m}$. 
A

Tie2-Plxnd1 Tg construct

\begin{tabular}{|c|c|c|c|}
\hline Tie2-Promoter & Plxnd1-cDNA & PolyA & Tie2-Enhancer \\
\hline $2.1 \mathrm{~kb}$ & $5.77 \mathrm{~kb}$ & $10 \mathrm{~kb}^{-}$ \\
\hline
\end{tabular}

B

Transgenic mice founder (F0)

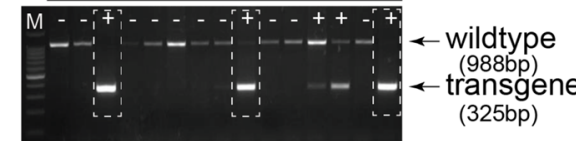

C

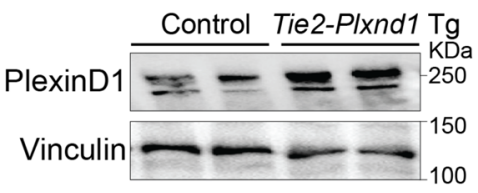

$\square$ Control

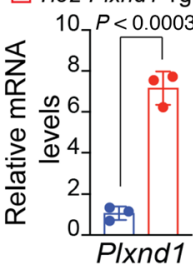

F Whole heart Right ventricle Left ventricle
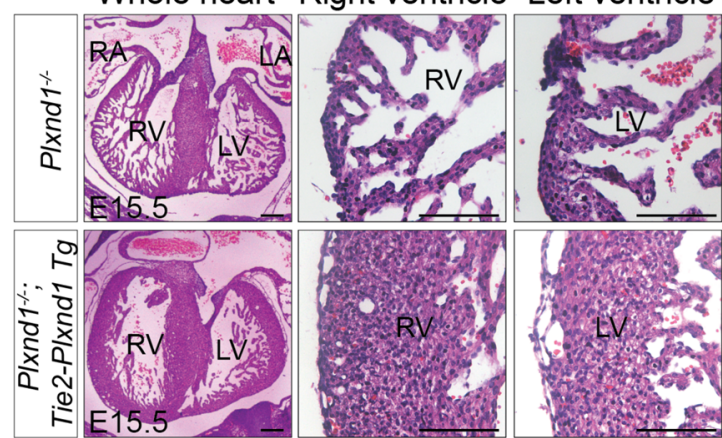

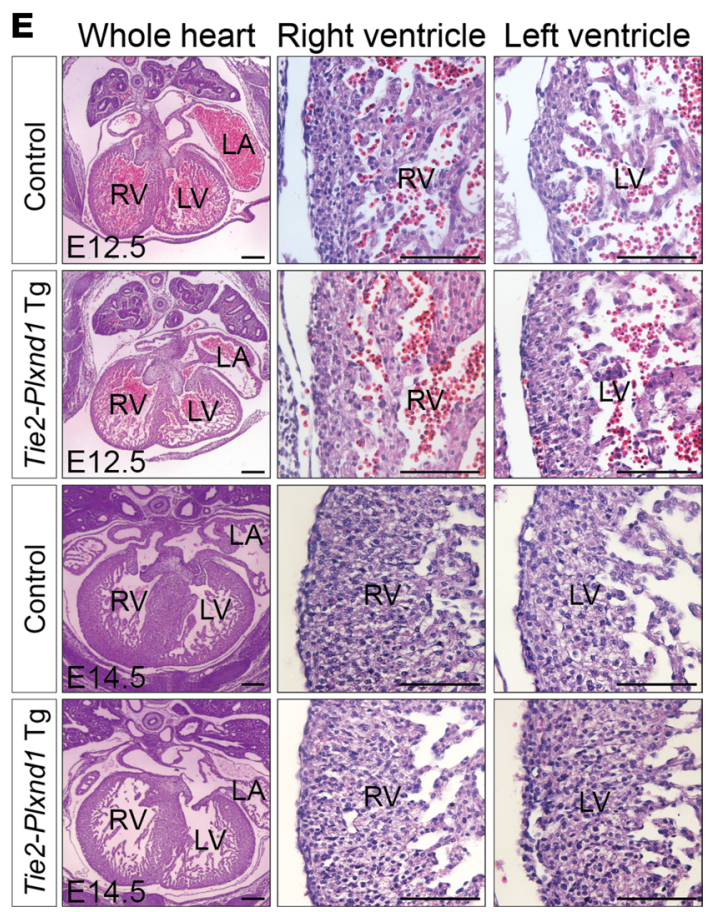

Figure 6. Endothelium-specific Plxnd1 overexpression does not affect cardiac chamber development but can rescue the hypertrabeculation and noncompaction defects when crossed to a PIxnd1/-- background. Scheme of the endothelial-specific Plxnd1-transgenic construct (A). Transgenic founder (FO) mice were identified through PCR screening. Three 325-bp transgene-positive founders (white dotted box) were used for generating F1 (B). Western blot analysis of PlexinD1 for F2 control (transgene negative) and Tie2-Plxnd1-Tg embryo lysate. Anti-vinculin antibody is used as a loading control (C). Realtime qPCR for PIxnd1 using RNA isolated from endothelial cells of E10.5 control and Tie2-PIxnd1-Tg embryo (D). A transgenic line showing elevated levels of Plexind1 proteins was used for subsequent experiments. H\&E staining of paraffin sections of E12.5 ( $n=5$ for each genotype) and E14.5 ( $n=6$ for each genotype) control and Tie2-PIxnd1-Tg hearts (E). H\&E staining of paraffin sections of E15.5 Plxnd1 1/- and Plxnd1 ${ }^{-/-}$Tie2-Plxnd1-Tg hearts (F). ( $n=4$ for each genotype). LA, left atrium; LV, left ventricle; RA, right atrium; RV, right ventricle. Scale bars: $100 \mu \mathrm{m}$.

To determine whether Sema3E has any role in myocardial trabeculation and the compaction process, we generated control and Sema3e $e^{-1-}$ embryos and performed histological and quantitative analyses using hearts isolated from E12.5 and E15.5. Histological analysis revealed myocardial trabeculation and compaction defects in Sema3e $e^{--}$embryos (Figure 7, D-G). At E12.5, the trabecular layer thickness was not significantly different between Sema3e $e^{-1-}$ mutant and control embryos. However, the compact myocardial layer thickness was significantly reduced in Sema3e $e^{-1}$ embryos. (Figure 7, D and E). Compared with control, the compact myocardial layer was significantly thin, and the trabecular layer was significantly thick in Sema3e $e^{-1}$ embryos at E15.5 (Figure 7, F and G). In addition to ventricular walls, we also observed noncompaction in the ventricular septal myocardium. However, in contrast to a fully penetrant hypertrabeculation and noncompaction phenotypes in

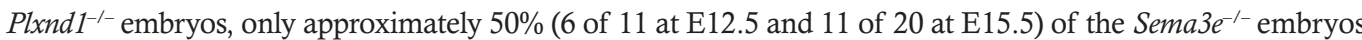
have these defects. Similar to the phenotypes seen in other class 3 Sema-knockout mice, the genetic background of the mice might have played a role in the incomplete penetrance in our current study (54).

\section{Discussion}

LVNC is one of the most characterized noncompaction cardiomyopathies. In children with all types of primary cardiomyopathy, LVNC is the third-most common cardiomyopathy after dilated cardiomyopathy and hypertrophic cardiomyopathy (55). LVNC can either occur in isolation or is associated with other congenital heart diseases, resulting in cardiac dysfunction. In LVNC cases, $22.6 \%$ are from LVNC alone, while 

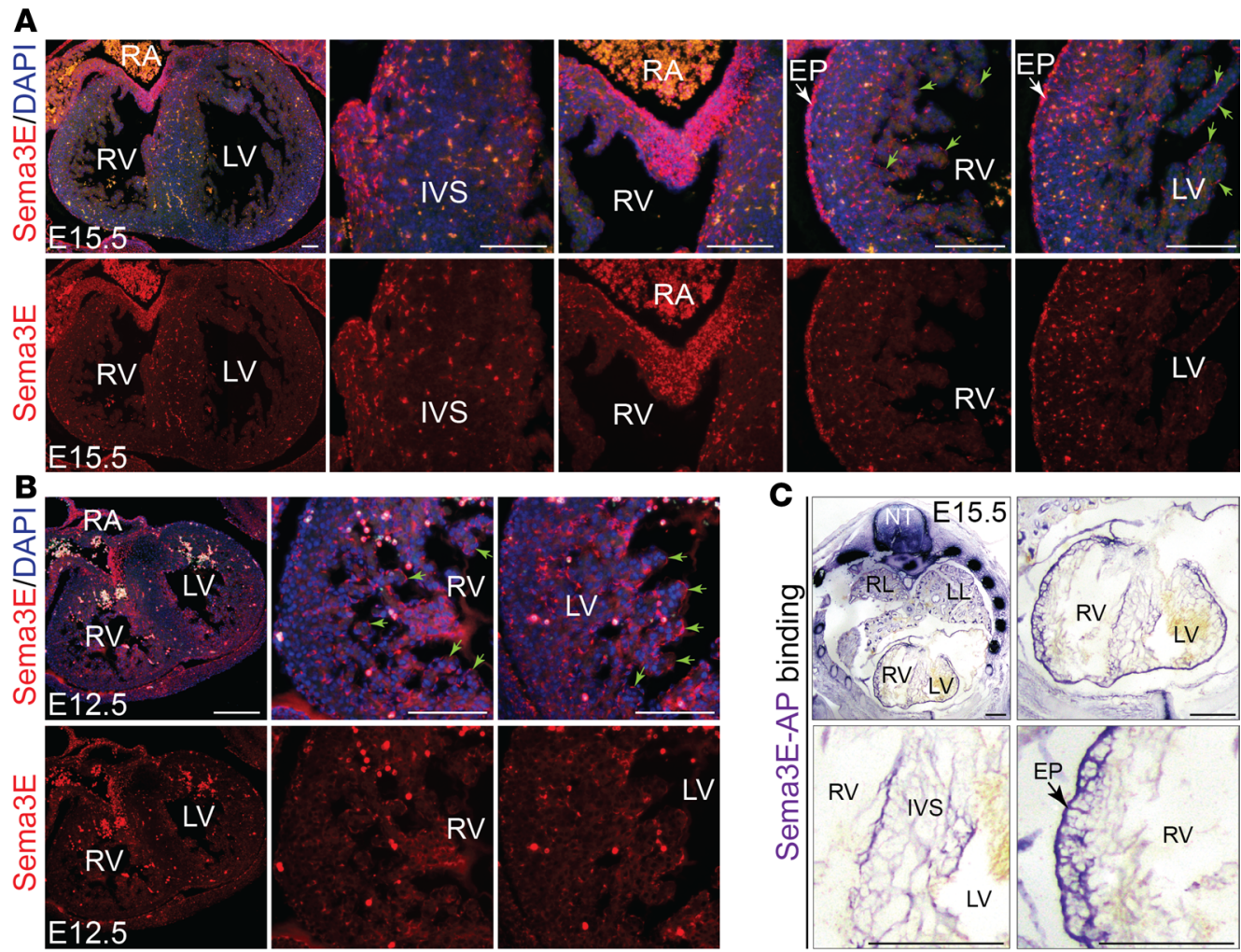

D Whole heart Right ventricle Left ventricle Ventricular septum E

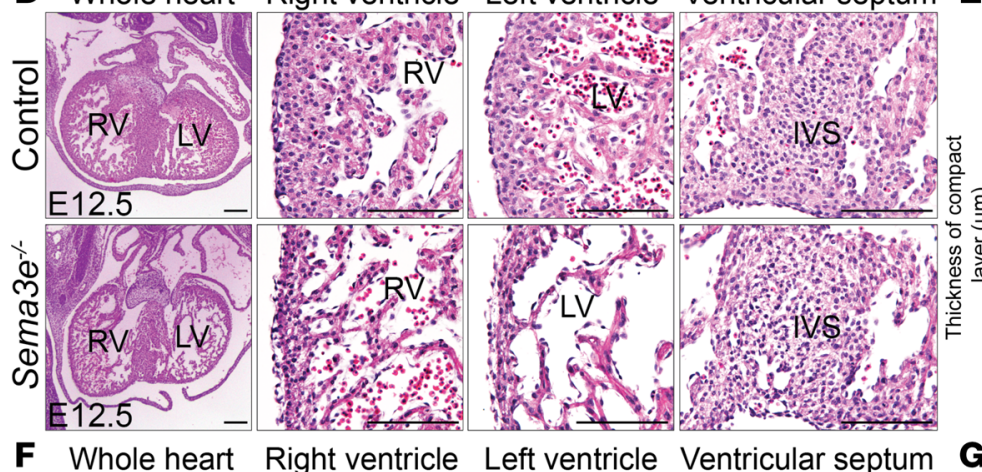

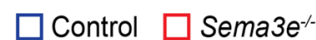

$$
\text { 吾 }
$$
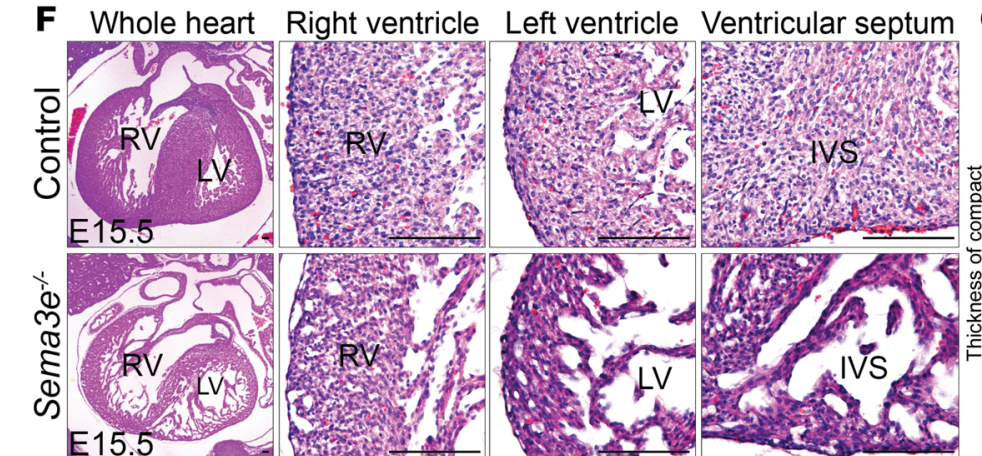

\section{G}

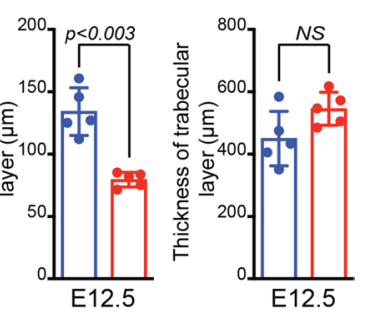

$\square$ Control $\square$ Sema3e-r $^{-12}$

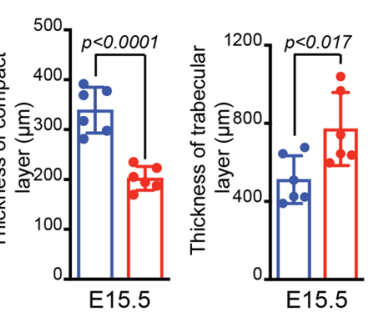

Figure 7. Sema3E is expressed in the heart and required for myocardial trabeculation and compaction. Immunostaining for Sema3E was performed on (A) E15.5 and (B) E12.5 WT heart sections. We have aligned 2 individual images to cover the whole heart at $\mathbf{A}$. Green arrows indicate Sema3E expression in endocardial cells. Scale bars: $200 \mu \mathrm{m}$. Sema3E-AP binding on transverse cryosections from E15.5 WT embryo. Higher magnification of heart, right ventricle wall, and interventricular septum are presented. Scale bars: $200 \mu \mathrm{m}$. (C) H\&E staining of paraffin sections of E12.5 and ( $\mathbf{D}$ and E) E15.5 (F and $\mathbf{G} ; n=10$ for each genotype) control ( $n=10$ at E12.5 and $n=17$ at E15.5) and Sema3e $e^{-/-}(n=11$ at E12.5 and $n=20$ at E15.5) hearts. Scale bars: $100 \mu \mathrm{m}$. Quantification of the thickness of the compact and trabecular layer in control ( $n=5$ at E12.5 and $n=6$ at E15.5) and Sema3e $e^{-/-}(n=5$ at E12.5 and $n=$ 6 at E15.5) hearts at different time points are indicated as E and G. EP, epicardium; IVS, interventricular septum; LL, left lung; LV, left ventricle; NT, neural tube; RA, right atrium; RL, right lung; RV, right ventricle. 
the rest are associated with dilated cardiomyopathy (58.7\%), hypertrophic cardiomyopathy (11\%), and indeterminate cardiomyopathy phenotype $(7.7 \%)(55,56)$. In fact, the most common congenital conditions associated with LVNC are right-sided lesions - especially Ebstein's anomaly, stenotic lesions of the left ventricular outflow tract, and tetralogy of Fallot (57-59).

LVNC is a clinically and genetically complex cardiomyopathy. Although multiple cellular and molecular mechanisms responsible for cardiac chamber development have been widely studied, various molecules and signaling pathways underlying LVNC have not been characterized. In this study, we reveal the role of the Sema3E/PlexinD1 signaling pathway in ventricular trabeculation and compaction using 4 different mouse models: Plxnd1-knockout, endothelium-specific Plxnd1-knockout, transgenic mice overexpressing Plxnd1 in endothelium, and Sema3e-knockout mice. For the first time, we show that Sema3E is expressed in the heart and is required for proper myocardial compaction. Mice with impaired Sema3E/PlexinD1 signaling develop the LVNC phenotype. Our results demonstrate that Sema3E/PlexinD1 signaling plays a critical role in ventricular chamber development by modulating ECM dynamics and the Notch signaling pathway.

The Notch signaling pathway is an evolutionarily conserved, intercellular signaling system that modulates cardiomyocyte proliferation and hence compaction and trabeculation (60). The Notch receptor Notch1 and its ligand Delta 4 are expressed in the endocardium throughout compaction. Notch1 promotes cardiomyocyte proliferation and differentiation by activating Efnb2 and Nrg1 expression within the endothelial cells and Bmp10 in the underlying trabeculated myocardium (3). Mutants of the Notch signaling pathway have trabeculation defects due to abnormal myocardial cell proliferation and differentiation, which leads to cardiac insufficiency and embryonic death in the mutants $(3,61)$. Consistently, loss-of-function mutation in either Nrg1 (or its receptors, ErbB2 or ErbB4) results in severe trabeculation defects and embryonic lethality (62-64). Cardiomyocyte proliferation defects seen in the Notch signaling pathway mutants can be rescued by exogenous Bmp10 alone or Bmp10 together with Nrg1 (3).

In our study, we found that Plxnd1-knockout mutants have hypertrabeculation and myocardial noncompaction, a characteristic LVNC phenotype. Molecular investigation of these mutants reveals that $P l x$ $n d 1$ deletion elevates the expression of Notch pathway-associated genes including Notch1, its downstream effector Nrg1, its receptor ErbB2, Hrt1/2/3 and EphrinB2/B4. These findings are consistent with a recent report by Kim et al. demonstrating that the Notch pathway is downstream of the Sema3E/PlexinD1 signaling pathway in endothelial cells (65). Our results also complement existing studies that show elevated Notch signaling in endocardial cells is associated with ventricular hypertrabeculation and noncompaction. For example, Mysliwiec et al. elucidated that Jarid2 is required in the endothelial cells to repress the Notch signaling pathway. Genetic deletion of Jarid2 in the endothelial cells leads to increased Notch1 activity in endocardial cells, causing hypertrabeculation and noncompaction of the ventricle (28). A study by Chen et al. demonstrated that Fkbpla is a negative modulator of Notch1 activity in endocardial cells. Loss of Fkbpla in the endothelial cells leads to ventricular hypertrabeculation and noncompaction (26). Another study by Yang et al. showed that increased Notch2 activity in Numb/Numb-like double mutants leads to increased Bmp10 expression causing hypertrabeculation and noncompaction defects (29).

Most recently, Lin et al. found that zinc transporter Slc39a8 is essential for cardiac ventricular compaction. Genetic deletion of Slc39a8 leads to increased Notch1 activity and Bmp10 expression, causing excessive trabeculation and myocardial noncompaction (27). These studies correlate both Notch pathway and Bmp10 expression with ventricular trabeculae formation. Although mice with Bmp10 overexpression display hypertrabeculation, Bmp10-deficient mice die in utero due to hypotrabeculation of the ventricular walls $(24,25)$. Expressions of Bmp10 and other cardiac chamber regulators including Irx3, Irx5, and PEG1 were upregulated in the Plxnd1 mutant hearts. Consistently, we also observed an increased trabecular cardiomyocyte proliferation in these mutants. These indicate that the Sema3E/PlexinD1 signaling pathway is upstream of Bmp10 and other regulators of trabeculae formation during myocardial compaction. Following inhibition of Notch signaling in Plxnd1 mutants, Notch1 and Bmp10 expression levels were back to basal supporting our conclusions. Collectively, these results imply that the resultant LVNC phenotype in Plxnd1 mutants is due to upregulation of the downstream Notch pathway and Bmp10 signaling.

Another phenomenon regulating cardiac chamber remodeling is the dynamic formation and degradation of the ECM (cardiac jelly). Both formation and degradation of the ECM is vital for the development and maturation of the cardiac chamber, respectively. Here, we demonstrate that ECM synthesis genes are strongly expressed, whereas the ECM proteolytic genes are either weakly expressed or repressed during the 
early phases of trabeculation (when ECM is required). On the other hand, when the ECM is not required during later stages of trabeculation, ECM synthesis genes are weakly expressed and ECM proteolytic genes are either highly expressed or derepressed. In Plxnd1 mutant embryos, immunohistochemical analysis exhibits excessive ECM accumulation. Quantitative analysis of ECM proteolytic and synthesis genes reveals that although Plxnd1 deletion did not alter expression of ECM synthesis genes, ECM proteolytic gene expression was decreased including Adamts metalloproteinases. Recently, many studies have described the role of ECM in proper cardiac chamber development and maturation. Hearts from mice deficient in Has 2 or Vcan genes required for ECM synthesis do not contain trabeculae $(32,33)$. Genes encoding metalloproteinases, including Adamts family members and MMPs, mediate ECM degradation and are essential for proper cardiac development. Both Adamts1- and Adamts9-deficient mice exhibit excessive ECM accumulation that results in ventricular noncompaction/hypertrabeculation defects $(34,35)$. Brg1, a chromatin remodeling factor, has been shown to inhibit Adamts1 expression in endocardial cells required for modulating trabeculation (35). The cerebral cavernous malformation pathway inhibits expression of Adamts 4 and Adamts 5 proteases that degrade cardiac jelly (36). A recent study by Del Monte-Nieto et al. showed that both Notch1 and Nrg1 signaling pathways critically regulate the cardiac jelly dynamics during the initial stages of trabeculation. Although signals from the myocardium control ECM synthesis, endocardial signals control ECM degradation (4). Lin et al. demonstrated that Zinc transporter Slc39a8 positively regulates Adamts1 expression and mice deficient for Slc39a8 developed the LVNC phenotype due to impaired ECM degradation (27). Consistent with these studies, our results suggest that hypertrabeculation and noncompaction in Plxnd1-knockout mice occur from excessive ECM accumulation resulting from decreased expression of ECM proteolytic genes. These findings also indicate that Sema3E/PlexinD1 signaling promotes ECM degradation. However, further investigation is required to confirm whether Sema3E/plexinD1 modulation of Notch and Nrg1-Erb2/4 signaling causes variation in the expression of ECM proteolytic genes in Plxnd1 mutants.

We observed that endothelium-specific Plxnd1-knockout mutants recapitulate the hypertrabeculation and noncompaction phenotypes of Plxnd1-knockout mice. This finding signifies that endothelial-specific Sema3E/PlexinD1 signaling regulates the cardiac remodeling process. We validated our hypothesis in experiments that restored Plxnd1 expression. Although endothelial-specific overexpression of Plxnd1 does not affect normal cardiac chamber formation, endothelial-specific restoration of Plxnd1 rescues hypertrabeculation and noncompaction defects. Therefore, Plxnd1 expression in the endocardium is essential for ventricular trabeculation and compaction.

We also demonstrate that Sema3E binds to the neural tube, lungs, cartilage, and heart. This finding indicates that Sema3E interacts with its receptor in these tissues to enable downstream signaling. In the heart, Sema3EAP binding is not restricted to the endothelial cells. This observation suggests that in addition to PlexinD1, Sema3E may bind to other receptors. Finally, we show that Sema3e mutants have thick trabeculae and thin myocardium, suggesting noncompaction and hypertrabeculation phenotypes. Together, these results reemphasize that the Sema3E/plexinD1 pathway plays a crucial role in ventricular trabeculation and compaction.

In summary, we showed that Sema3E is expressed in the heart, and in association with its receptor PlexinD1, it regulates Notch pathway genes, regulators of cardiomyocyte proliferation, and expression of ECM proteolytic genes. These genes participate in ventricular trabeculation and the compaction process. Hence, our work unveils that the Sema3E/plexinD1 pathway governs the process of cardiac trabeculation and myocardial noncompaction. Additional investigations are necessary to further associate our molecular findings with the LVNC phenotype. Nonetheless, our findings provide a new insight into the pathogenesis of LVNC. In humans, de novo mutations in PLXND1 are associated with cardiac and neuronal defects. However, it is not clear whether these mutations are associated with cardiac chamber maturation (66, 67). Because our analytical investigations validate that Sema3E/PlexinD1 signaling plays a critical role in regulating myocardial trabeculation and compaction, we suggest that Sema3e and Plxnd1 can be used as candidate biomarkers for screening patients with LVNC who have these genetic mutations. Altogether, our work provides new insights on heart morphogenesis that can potentially resolve the complexity of LVNC.

\section{Methods}

Animal experiments. Plxnd1 ${ }^{+/-}, P l x n d 1^{f l / f l}$, Sema3e $e^{+/-}$, and Tie $2^{\text {Cre }}$ mice have been previously described $(38,49,50)$.

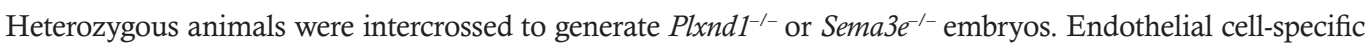
$P l x n d 1$ mutant mice were generated by crossing the Tie $2^{\mathrm{Cre} /+}$ mice with $P l x n d l^{f / f l}$ mice. Resulting Tie $2^{\mathrm{Cre} /+} P l x$ $n d 1^{f l+}$ offspring were then back-crossed to $P l x n d 1^{f / f l}$ mice to obtain Tie ${ }^{\text {Crel+}} P l x n d 1^{f l f l}$ (presented as Plxnd1 $1^{\text {eKO }}$ 
throughout) mice. To obtain mice overexpressing Plxnd1 in endothelial cells, a mice tyrosine kinase receptor Tie2 promoter was used. The coding sequence for the murine Plxnd1 gene was amplified and inserted into the expression vector containing the $2 \mathrm{~kb}$ Tie 2 promoter together with 10-kb Tie 2 enhancer originated from intron-1 of the endogenous murine Tie 2 gene. The targeting vector, Tie2-Plxnd1-Tg was linearized and microinjected into fertilized eggs prepared from superovulated C57BL/6 mice. The eggs were transferred into the oviducts of pseudopregnant foster mothers. Founder mice were identified by PCR analysis using PXDgt1 (forward): 5'-ACCAACAAGCTTCTGTACGCGAAGGAG-3' and PXDgt2 (reverse): 5'-ATCAGGCCTCGCTGTAACACTCATAG-3' primers. Three lines of the Plxnd1 transgene in the C57BL/6 background were established and used for the analysis. Tie2-Plxnd1-Tg mice were generated at the Biological Resource Centre, ASTAR, Singapore. For rescue experiments, Tie2-Plxnd1- Tg mice were mated with $P l x n d 1^{+/-}$mice to generate Tie2-Plxnd1 Tg Plxnd1 ${ }^{+/-}$mice. Then, Tie2-Plxnd1-Tg Plxnd1 ${ }^{+-}$mice were time mated with Plxnd1 ${ }^{+/-}$mice to generate Tie2-Plxnd1-Tg Plxnd1 ${ }^{-/-}$mice. Control $\left(P l x n d 1^{+/+}\right.$or $P l x n d 1^{+/-}$or Sema3e $e^{+/+}$or Sema3e $e^{+/-}$or Plxnd1 $1^{f l f l}$ or $P l x n d 1^{f /+}$ or Tie $\left.2^{\mathrm{Cre} /+} P l x n d 1^{f /+}\right)$ and mutant $\left(P l x n d 1^{-/-}\right.$or Sema3e $e^{-/-}$or Tie $2^{\mathrm{Cre} /+}$ Plxnd $\left.1^{f / f f}\right)$ embryos were harvested from timed pregnancies counting the afternoon of the plug date as E0.5. Embryos were dissected in PBS and fixed in 4\% paraformaldehyde solution in PBS. Genotyping was performed on DNA isolated from yolk sacs or tail biopsies. Littermate embryos were analyzed in all experiments unless otherwise noted.

Histology and immunohistochemistry. Histology and immunohistochemistry were performed as described previously (68-71). Briefly, embryos were harvested at selected time points and fixed overnight in 4\% paraformaldehyde. Subsequently, the samples were dehydrated in various percentages of ethanol, processed for paraffin embedding and sectioned at $10 \mu \mathrm{m}$. The sections were mounted on slides, deparaffinized, and hydrated followed by blocking of endogenous peroxidase activity with $3 \% \mathrm{H}_{2} \mathrm{O}_{2}$ for 2 hours at room temperature. Next, sections were incubated with primary antibodies overnight at $4^{\circ} \mathrm{C}$. Sections were then washed with tris-buffered saline with Tween 20 and incubated with a secondary antibody at room temperature for 2 hours. Thereafter, HRP activity was detected either by using a DAB kit (Vector Laboratories, catalog SK-4100) or TSA kit (Perkinelmer Singapore Pte Ltd, catalog NEL760001KT). Sections were then dehydrated and mounted with DPX Mountant for observation under the light or a fluorescent Lieca microscope. Sections incubated with Alexa Fluor secondary antibodies were directly observed under fluorescent microscope after washing and mounting. Embryos from 4-6 mice from each group were collected and at least 5 different regions of each section were studied. All images were quantified using Image J software and the thickness of ventricular walls was analyzed using GraphPad Prism.

Western blotting. Western blotting studies were performed to examine protein levels. Snap-frozen E12.5 and E14.5 heart tissues were pooled (3-4 hearts for each genotype) and homogenized by using RIPA lysis and extraction buffer (Thermo Fisher Scientific, catalog 89901) along with protease (MilliporeSigma, catalog P8340) and phosphatase inhibitors (Thermo Fisher Scientific, catalog 88667). Equal amounts of protein $(30-40 \mu \mathrm{g})$ were loaded in $6 \%-10 \%$ SDS-PAGE gels. After electrophoresis, proteins were transferred onto PVDF membranes and incubated overnight with their respective antibodies. Blots were developed using WEST-Queen Western Blot Detection System (Intron Biotechnology, catalog 16026).

Proliferation assay. To determine the changes in cell proliferation, Ki-67 and MF20 double immunostaining was performed on E14.5 control and $P l x n d 1^{-/-}$heart sections as previously described (68, 72). DAPI (Vector Laboratories) was used to stain nuclei. For each genotype, images of 4-6 different sections of 3-4 independent embryos were used. The total number of proliferating cells was manually counted on each section and analyzed using GraphPad Prism.

Antibodies. The following antibodies were used for Western blotting and immunohistochemistry: Notch1 (Santa Cruz, catalog sc-6014), Cleaved Notch1 (Val1744) (Cell Signaling, catalog 4147S), ErbB2 (Cell Signaling, catalog 2242), pErbB2 (Cell Signaling, catalog 2247), Bmp10 (R\&D System, catalog MAB6038), Vinculin (MilliporeSigma, catalog V9131), Gapdh (Santa Cruz, catalog sc-20357), Actin (Santa Cruz, catalog sc-47778), MF20-C (DSHB), Ki-67 (Abcam, catalog ab15580), Sema3E (MilliporeSigma, catalog HPA029419), Varsican (Thermo Fisher Scientific, catalog PA11748A), Adamts1 (Santa Cruz, catalog sc-47727), Fibronectin (Santa Cruz, catalog sc-8422), and PlexinD1 (R\&D System, catalog AF4160).

$R N A$ extraction and quantitative RT-PCR. Total RNA was isolated from embryonic hearts using TRIzol (Life Technologies, catalog 15596-018). cDNA synthesis was performed with 1- $\mu$ g RNA using random hexamers and the SuperScript-III First-Strand Synthesis system (Life Technologies, catalog 18080-051). Samples were treated with DNaseI before reverse transcription to remove any DNA contamination (Thermo Fisher Scientific, catalog 18068015). Gene expression was measured by quantitative RT-PCR using HOT FIREPol EvaGreen qPCR 
supermix. All qPCRs were conducted in 384 plates using the 7900HT Sequence Detection System and the results were analyzed using SDS2.0 software (Applied Biosystem). Both the signals and relative gene expression were normalized to corresponding Gapdh controls. Endothelial cell isolation was performed as described previously (41). RNA isolation, cDNA synthesis, and qPCR were performed as described before.

Notch pathway inhibition. For in vivo notch pathway inhibition, the $\gamma$-secretase inhibitor DBZ (Tocris Bioscience, catalog 4489$)$ was given by i.p. injection at 11.5 days of pregnancy $(10 \mu \mathrm{mol} / \mathrm{kg})$. Control mice were administered with vehicle (0.5\% hydroxypropyl methylcellulose in $0.1 \%$ Tween 20$)$. Embryos were harvested at E13.5 and subjected to morphological, histological, and molecular analysis.

Alkaline phosphatase binding assay. The Sema3E-AP binding assay was performed according to the published protocol (38). Briefly, WT embryos were dissected in PBS and frozen immediately in liquid nitrogen, then stored at $-80^{\circ} \mathrm{C}$ until use. Cryosections were prepared, fixed in ice-cold methanol for 8 to 10 minutes and then washed 3 times ( 5 min each) with washing buffer $\left(1 \times \mathrm{PBS}+4 \mathrm{mM} \mathrm{MgCl}_{2}\right)$. To reduce nonspecific binding, sections were incubated in blocking buffer (PBS $+4 \mathrm{mM} \mathrm{MgCl}_{2}+10 \% \mathrm{FBS}$ ) for $45-60$ minutes. After blocking, sections were incubated with binding solution (2-nM AP-tagged Sema3E diluted with PBS $+4 \mathrm{mM} \mathrm{MgCl}_{2}$ and buffered with 0.5 M HEPES, pH 7) in a humidified chamber for 2 hours at room temperature. Sections were washed 5 times in washing buffer and fixed with a fixative solution ( $60 \%$ acetone, $1 \%$ formaldehyde, $20 \mathrm{mM} \mathrm{HEPES,} \mathrm{pH} \mathrm{7)} \mathrm{for}$ 2 minutes. Sections were washed 3 times in PBS and then heat inactivated for 2 hours at $65^{\circ} \mathrm{C}$ using a water bath to reduce endogenous alkaline phosphatase activity. Sections were washed twice in PBS, incubated in AP buffer (100 mM Tris- $\mathrm{HCl} \mathrm{pH} 9.5,100 \mathrm{mM} \mathrm{NaCl}, 5 \mathrm{mM} \mathrm{MgCl}_{2}$ ) for 5 minutes and then incubated overnight in the developing solution (AP buffer with nitro-blue tetrazolium chloride and 5-bromo-4-chloro-3'-indolyphosphate p-toluidine. On the next day, sections were washed again in PBS and mounted for imaging.

Statistics. Statistical analyses were performed using the 2-tailed Student's $t$ test. Data were expressed as mean $\pm \mathrm{SD}$. Differences were considered significant when $P<0.05$.

Study approval. All animal procedures were approved by the Institutional Animal Care and Use Committee at Duke-NUS Medical School/Singhealth conforming to the Guide for the Care and Use of Laboratory Animals (National Academies Press, 2011).

\section{Author contributions}

RS, DMC, AS, PG, NT, and MKS performed experiments and analyzed data. AU and JAE provided reagents. MKS oversaw the entire project, designed the experiments, and wrote the article. All authors discussed the results and implications and commented on the manuscript at all stages.

\section{Acknowledgments}

This work was supported by grants from Duke-NUS Medical School Singapore, the Goh foundation, and Singapore Ministry of Health's National Medical Research Council (MOH-OFIRG18nov-0005) to MKS. We would like to thank Shamini Guna Shekeran and Masum M. Mia for technical assistance.

Address correspondence to: Manvendra K. Singh, Program in Cardiovascular \& Metabolic Disorders, Duke-NUS Medical School, 8 College Road, Office 08-15, Singapore 169857. Phone: 65.66013098; Email: manvendra.singh@duke-nus.edu.sg.

1. Sedmera D, Pexieder T, Vuillemin M, Thompson RP, Anderson RH. Developmental patterning of the myocardium. Anat Rec. 2000;258(4):319-337.

2. Sedmera D, Thomas PS. Trabeculation in the embryonic heart. Bioessays. 1996;18(7):607.

3. Grego-Bessa J, et al. Notch signaling is essential for ventricular chamber development. Dev Cell. 2007;12(3):415-429.

4. Del Monte-Nieto G, et al. Control of cardiac jelly dynamics by NOTCH1 and NRG1 defines the building plan for trabeculation. Nature. 2018;557(7705):439-445.

5. Samsa LA, Yang B, Liu J. Embryonic cardiac chamber maturation: trabeculation, conduction, and cardiomyocyte proliferation. Am J Med Genet C Semin Med Genet. 2013;163C(3):157-168.

6. Risebro CA, Riley PR. Formation of the ventricles. Scientific World Journal. 2006;6:1862-1880.

7. Pasumarthi KB, Field LJ. Cardiomyocyte cell cycle regulation. Circ Res. 2002;90(10):1044-1054.

8. Icardo JM, Fernandez-Terán A. Morphologic study of ventricular trabeculation in the embryonic chick heart. Acta Anat (Basel). 1987;130(3):264-274.

9. Anderson RH, Sanchez-Quintana D, Niederer P, Lunkenheimer PP. Structural-functional correlates of the 3-dimensional arrangement of the myocytes making up the ventricular walls. J Thorac Cardiovasc Surg. 2008;136(1):10-18.

10. Jenni R, Oechslin E, Schneider J, Attenhofer Jost C, Kaufmann PA. Echocardiographic and pathoanatomical characteristics of isolated left ventricular non-compaction: a step towards classification as a distinct cardiomyopathy. Heart. 2001;86(6):666-671. 
tion. Cardiol Young. 2005;15(4):345-364.

12. Towbin JA. Left ventricular noncompaction: a new form of heart failure. Heart Fail Clin. 2010;6(4):453-69, viii.

13. Oechslin E, Jenni R. Left ventricular non-compaction revisited: a distinct phenotype with genetic heterogeneity? Eur Heart $J$. 2011;32(12):1446-1456.

14. Fazio G, et al. Noncompaction of the right ventricle. Pediatr Cardiol. 2010;31(4):576-578.

15. Ranganathan A, Ganesan G, Sangareddi V, Pillai AP, Ramasamy A. Isolated noncompaction of right ventricle — a case report Echocardiography. 2012;29(7):E169-E172.

16. Tigen K, Karaahmet T, Gurel E, Cevik C, Basaran Y. Biventricular noncompaction: a case report. Echocardiography. 2008;25(9):993-996.

17. Bhatia NL, Tajik AJ, Wilansky S, Steidley DE, Mookadam F. Isolated noncompaction of the left ventricular myocardium in adults: a systematic overview. J Card Fail. 2011;17(9):771-778.

18. Ichida F, et al. Clinical features of isolated noncompaction of the ventricular myocardium: long-term clinical course, hemodynamic properties, and genetic background. J Am Coll Cardiol. 1999;34(1):233-240.

19. Paterick TE, Tajik AJ. Left ventricular noncompaction: a diagnostically challenging cardiomyopathy. Circ J. 2012;76(7):1556-1562

20. Zaffran S, Frasch M. Early signals in cardiac development. Circ Res. 2002;91(6):457-469.

21. Bruneau BG. Signaling and transcriptional networks in heart development and regeneration. Cold Spring Harb Perspect Biol. 2013;5(3):a008292.

22. Zhang K, et al. A Notch1-neuregulin1 autocrine signaling loop contributes to melanoma growth. Oncogene. 2012;31(43):4609-4618.

23. Del Monte G, Grego-Bessa J, González-Rajal A, Bolós V, De La Pompa JL. Monitoring Notch1 activity in development: evidence for a feedback regulatory loop. Dev Dyn. 2007;236(9):2594-2614.

24. Chen H, et al. BMP10 is essential for maintaining cardiac growth during murine cardiogenesis. Development. 2004;131(9):2219-2231

25. Chen H, Zhang W, Li D, Cordes TM, Mark Payne R, Shou W. Analysis of ventricular hypertrabeculation and noncompaction using genetically engineered mouse models. Pediatr Cardiol. 2009;30(5):626-634.

26. Chen $\mathrm{H}$, et al. Fkbp1a controls ventricular myocardium trabeculation and compaction by regulating endocardial Notch1 activity. Development. 2013;140(9):1946-1957.

27. Lin W, et al. Zinc transporter Slc39a8 is essential for cardiac ventricular compaction. J Clin Invest. 2018;128(2):826-833.

28. Mysliwiec MR, Bresnick EH, Lee Y. Endothelial Jarid2/Jumonji is required for normal cardiac development and proper Notch1 expression. J Biol Chem. 2011;286(19):17193-17204

29. Yang J, et al. Inhibition of Notch2 by Numb/Numblike controls myocardial compaction in the heart. Cardiovasc Res. 2012;96(2):276-285

30. Zhang W, Chen H, Qu X, Chang CP, Shou W. Molecular mechanism of ventricular trabeculation/compaction and the pathogenesis of the left ventricular noncompaction cardiomyopathy (LVNC). Am J Med Genet C Semin Med Genet. 2013;163C(3):144-156

31. Lockhart M, Wirrig E, Phelps A, Wessels A. Extracellular matrix and heart development. Birth Defects Res Part A Clin Mol Teratol. 2011;91(6):535-550.

32. Camenisch TD, et al. Disruption of hyaluronan synthase-2 abrogates normal cardiac morphogenesis and hyaluronan-mediated transformation of epithelium to mesenchyme. J Clin Invest. 2000;106(3):349-360.

33. Hatano S, et al. Versican/PG-M is essential for ventricular septal formation subsequent to cardiac atrioventricular cushion development. Glycobiology. 2012;22(9):1268-1277.

34. Kern CB, et al. Reduced versican cleavage due to Adamts9 haploinsufficiency is associated with cardiac and aortic anomalies. Matrix Biol. 2010;29(4):304-316.

35. Stankunas K, et al. Endocardial Brg1 represses ADAMTS1 to maintain the microenvironment for myocardial morphogenesis. Dev Cell. 2008;14(2):298-311.

36. Zhou Z, et al. The cerebral cavernous malformation pathway controls cardiac development via regulation of endocardial MEKK3 signaling and KLF expression. Dev Cell. 2015;32(2):168-180

37. Epstein JA, Aghajanian H, Singh MK. Semaphorin signaling in cardiovascular development. Cell Metab. 2015;21(2):163-173.

38. Gu C, et al. Semaphorin 3E and plexin-D1 control vascular pattern independently of neuropilins. Science. 2005;307(5707):265-268

39. Degenhardt K, et al. Semaphorin 3d signaling defects are associated with anomalous pulmonary venous connections. Nat Med. 2013;19(6):760-765

40. Aghajanian H, Choi C, Ho VC, Gupta M, Singh MK, Epstein JA. Semaphorin 3d and semaphorin 3e direct endothelial motility through distinct molecular signaling pathways. J Biol Chem. 2014;289(26):17971-17979.

41. Katz TC, et al. Distinct compartments of the proepicardial organ give rise to coronary vascular endothelial cells. Dev Cell. 2012;22(3):639-650.

42. Meadows SM, Ratliff LA, Singh MK, Epstein JA, Cleaver O. Resolution of defective dorsal aortae patterning in Sema3E-deficient mice occurs via angiogenic remodeling. Dev Dyn. 2013;242(5):580-590

43. Zygmunt T, et al. Semaphorin-PlexinD1 signaling limits angiogenic potential via the VEGF decoy receptor sFlt1. Dev Cell. 2011;21(2):301-314

44. Toyofuku T, et al. Guidance of myocardial patterning in cardiac development by Sema6D reverse signalling. Nat Cell Biol. 2004;6(12):1204-1211

45. Bouvrée K, et al. Semaphorin3A, Neuropilin-1, and PlexinA1 are required for lymphatic valve formation. Circ Res. 2012;111(4):437-445.

46. Takamatsu H, et al. Semaphorins guide the entry of dendritic cells into the lymphatics by activating myosin II. Nat Immunol. 2010;11(7):594-600.

47. Takegahara N, et al. Plexin-A1 and its interaction with DAP12 in immune responses and bone homeostasis. Nat Cell Biol. 2006;8(6):615-622.

48. van der Zwaag B, et al. PLEXIN-D1, a novel plexin family member, is expressed in vascular endothelium and the central nervous system during mouse embryogenesis. Dev Dyn. 2002;225(3):336-343

49. Gitler AD, Lu MM, Epstein JA. PlexinD1 and semaphorin signaling are required in endothelial cells for cardiovascular development. Dev Cell. 2004;7(1):107-116. 
50. Zhang Y, et al. Tie2Cre-mediated inactivation of plexinD1 results in congenital heart, vascular and skeletal defects. Dev Biol. 2009;325(1):82-93.

51. Oh WJ, Gu C. The role and mechanism-of-action of Sema3E and Plexin-D1 in vascular and neural development. Semin Cell Dev Biol. 2013;24(3):156-162.

52. Zhao C, et al. Numb family proteins are essential for cardiac morphogenesis and progenitor differentiation. Development. 2014;141(2):281-295.

53. Baardman ME, et al. Common arterial trunk and ventricular non-compaction in Lrp2 knockout mice indicate a crucial role of LRP2 in cardiac development. Dis Model Mech. 2016;9(4):413-425.

54. Feiner L, et al. Targeted disruption of semaphorin 3C leads to persistent truncus arteriosus and aortic arch interruption. Develop ment. 2001;128(16):3061-3070.

55. Lee TM, et al. Pediatric cardiomyopathies. Circ Res. 2017;121(7):855-873

56. Jefferies JL, et al. Cardiomyopathy phenotypes and outcomes for children with left ventricular myocardial noncompaction: results from the pediatric cardiomyopathy registry. J Card Fail. 2015;21(11):877-884.

57. Zuckerman WA, Richmond ME, Singh RK, Carroll SJ, Starc TJ, Addonizio LJ. Left-ventricular noncompaction in a pediatric population: predictors of survival. Pediatr Cardiol. 2011;32(4):406-412.

58. Stähli BE, et al. Left ventricular non-compaction: prevalence in congenital heart disease. Int J Cardiol. 2013;167(6):2477-2481.

59. Towbin JA, Lorts A, Jefferies JL. Left ventricular non-compaction cardiomyopathy. Lancet. 2015;386(9995):813-825.

60. MacGrogan D, Münch J, de la Pompa JL. Notch and interacting signalling pathways in cardiac development, disease, and regeneration. Nat Rev Cardiol. 2018;15(11):685-704.

61. Conlon RA, Reaume AG, Rossant J. Notch1 is required for the coordinate segmentation of somites. Development. 1995;121(5):1533-1545.

62. Lee KF, Simon H, Chen H, Bates B, Hung MC, Hauser C. Requirement for neuregulin receptor erbB2 in neural and cardiac development. Nature. 1995;378(6555):394-398.

63. Gassmann M, et al. Aberrant neural and cardiac development in mice lacking the ErbB4 neuregulin receptor. Nature. 1995;378(6555):390-394.

64. Meyer D, Birchmeier C. Multiple essential functions of neuregulin in development. Nature. 1995;378(6555):386-390.

65. Kim J, Oh WJ, Gaiano N, Yoshida Y, Gu C. Semaphorin 3E-Plexin-D1 signaling regulates VEGF function in developmental angiogenesis via a feedback mechanism. Genes Dev. 2011;25(13):1399-1411.

66. Tomas-Roca L, et al. De novo mutations in PLXND1 and REV3L cause Möbius syndrome. Nat Commun. 2015;6:7199.

67. Ta-Shma A, et al. Isolated truncus arteriosus associated with a mutation in the plexin-D1 gene. Am J Med Genet A. 2013;161A(12):3115-3120

68. Singh A, et al. Hippo signaling mediators Yap and Taz are required in the epicardium for coronary vasculature development Cell Rep. 2016;15(7):1384-1393.

69. Lee KY, et al. Tbx15 controls skeletal muscle fibre-type determination and muscle metabolism. Nat Commun. 2015;6:8054

70. Singh N, Gupta M, Trivedi CM, Singh MK, Li L, Epstein JA. Murine craniofacial development requires Hdac3-mediated repression of Msx gene expression. Dev Biol. 2013;377(2):333-344.

71. Greicius G, et al. pericryptal stromal cells are the critical source of Wnts and RSPO3 for murine intestinal stem cells in vivo. Proc Natl Acad Sci USA. 2018;115(14):E3173-E3181.

72. Singh MK, Lu MM, Massera D, Epstein JA. MicroRNA-processing enzyme Dicer is required in epicardium for coronary vasculature development. J Biol Chem. 2011;286(47):41036-41045. 